\title{
An unusual cause of shortness of breath
}

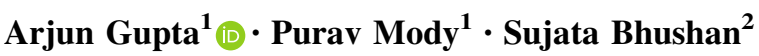

Received: 23 September 2015/ Accepted: 10 December 2015/Published online: 29 December 2015

(C) SIMI 2015

\section{Clinical case}

A 65-year-old man with a history of esophagitis, presented with low grade fever, poor appetite and a fall at home. Admission vitals were stable with temperature $37.9^{\circ} \mathrm{C}$ $\left(100.1^{\circ} \mathrm{F}\right)$. He was found to be dehydrated, and a urinalysis was compatible with urinary tract infection. An admission chest X-ray study was unremarkable. He was admitted for intravenous hydration and antibiotics. He was doing well after admission, when overnight he complained of new onset shortness of breath and epigastric pain. He reported that he had vomited approximately $150 \mathrm{~mL}$ of 'brownish material' 30 min prior, and his symptoms had started after that.

The physical examination was notable for tachycardia (heart rate 104 beats/min), tachypnea (respiratory rate 22 breaths/min), decreased breath sounds at bilateral lung bases, hypoxia (oxygen saturation $86 \%$ on $2 \mathrm{~L}$ nasal cannula), and epigastric tenderness. A chest X-ray study demonstrated pneumomediastinum and bilateral pleural effusions. A CT scan of the chest confirmed the presence of bilateral pnemuothoraces, pneumomediastinum and bilateral pleural effusions (Fig. 1).

Given the onset of symptoms after a bout of vomiting, the diagnosis of esophageal rupture was considered. Bilateral chest tubes were urgently placed. Primary open surgical repair was deferred. Upper gastrointestinal endoscopy showed necrotic tissue and a full thickness tear in the

Arjun Gupta

sujata.bhushan@va.gov

1 Department of Internal Medicine, University of Texas Southwestern Medical Centre, 5323 Harry Hines Blvd, Dallas, TX 75390-9047, USA

2 VA North Texas Health Care System, Dallas, TX, USA esophagus around a hiatal hernia. An esophageal stent was successfully placed, and broad spectrum antibiotics initiated with continued placement of bilateral chest tubes. Biopsy of the necrotic tissue showed chronic inflammation without evidence of malignancy. The stent was removed after 4 weeks at which time repeat upper gastrointestinal endoscopy revealed a healed tear. At this time, the patient was tolerating oral feeds, and he was discharged.

The esophagus lacks a serosal layer containing collagen and elastic fibers, making the wall weaker and more likely to rupture at lower pressures than the rest of the gastrointestinal tract [1]. Most cases of esophageal rupture are iatrogenic, and the term 'Boerhaave syndrome' is reserved for those induced spontaneously by uncoordinated vomiting [2].

The syndrome is characterized by full thickness tear, usually involving the posterolateral wall of distal esophagus, due to barogenic trauma induced by uncoordinated vomiting. This causes an acute rise in intraluminal pressure with esophageal disruption and forceful expulsion of gastric contents into the mediastinum or pleura with consequent chemical and bacterial mediastinitis. Mackler's classic triad of vomiting, chest pain and subcutaneous emphysema is seen in only $50 \%$ of cases. The physical examination reveals few specific signs. Subcutaneous emphysema, dullness to percussion (secondary to a pleural effusion), decreased breath sounds (due to pneumothorax), Hamman's sign (a crunch like noise over the precordium due to mediastinal air) are seen in $20-30 \%$ of patients. A chest X-ray study may show pneumomediastinum, subcutaneous emphysema, pleural effusions, pneumothorax, mediastinal widening, or hydropneumothorax. In patients with a suggestive clinical presentation, chest radiography, CT scan or esophagogram should be promptly obtained.

A CT scan allows rapid detection of minute amounts of air, and an esophagogram can pinpoint the lesion more 


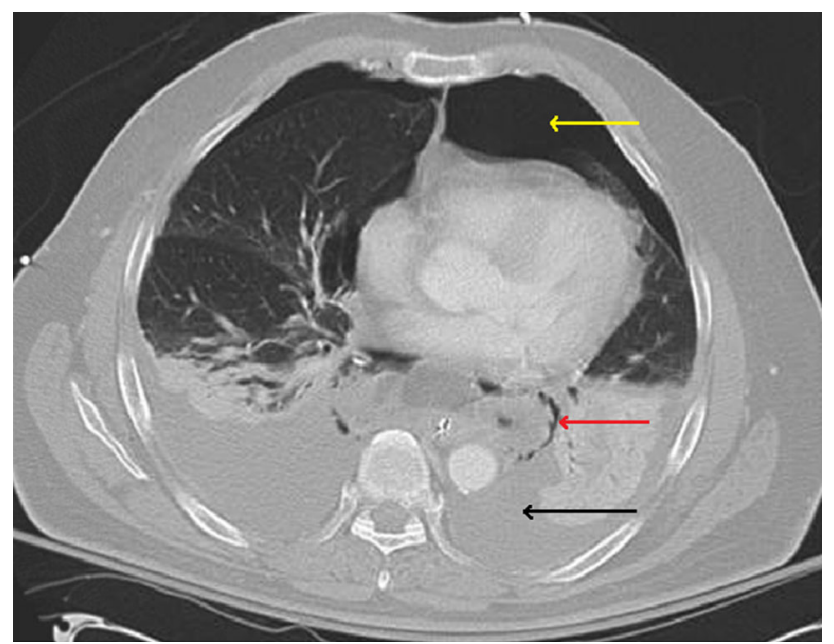

Fig. 1 Chest CT demonstrating the classic combination of pneumothorax (yellow arrow), pneumomediastinum (red arrow) and pleural effusion (black arrow), leading to a diagnosis of esophageal rupture (color figure online)

accurately. The role of upper endoscopy remains controversial as both the endoscope and insufflations of air can extend the perforation and worsen the pneumomediastinum. However, it can be a useful tool for diagnosis and treatment planning (as in our patient).

Management goals include early diagnosis, aggressive resuscitation, sepsis control, nutritional support and restoration of gastrointestinal continuity. Treatment depends upon the size and location of the perforation, whether it is contained, how rapidly it is diagnosed, any underlying esophageal pathology and the patient's associated co-morbidities. Conservative management can be considered with a contained perforation and includes continuous nasogastric suction, intravenous antibiotics, and drainage of pleural effusions. Surgery with primary repair or esophageal resection should be performed in patients with free perforation (within 12-24 h) whereas esophageal resection with cervical esophagostomy and gastrostomy is indicated in delayed presenters. An alternative approach with endoscopic placement of removable Polyflex stents or self-expandable metal stents (SEMS) has been shown to be a good alternative to surgery in early presenters with contained perforations [3].

\section{Compliance with ethical standards}

Conflict of interest The authors declare that they have no conflict of interest.

Statement of human and animal rights All procedures perfomed in human participants in this study were in accordance with the ethical standards of the institutional committee and with the 1964 Helsinki Declaration and its later amendments. This article does not contain any studies on animals performed by any author.

Informed consent Informed consent was obtained from the patient.

\section{References}

1. Jagminas L, Silverman R (1996) Boerhaave's syndrome presenting with abdominal pain and right hydropneumothorax. Am J Emerg Med 14(1):53-56

2. Khan AZ, Strauss D, Mason RC (2007) Boerhaave's syndrome: diagnosis and surgical management. Surgeon 5(1):39-44

3. Kiev J, Amendola M, Bouhaidar D et al (2007) A management algorithm for esophageal perforation: Am J Surg 194:103-106 